\title{
Testing the (generalized) Chaplygin gas model with the Lookback time-Redshift data
}

\author{
Zhengxiang $\mathrm{Li}^{1,2}$, Puxun $\mathrm{Wu}^{1,2}$ and Hongwei $\mathrm{Yu}^{1,2 *}$ \\ ${ }^{1}$ Department of Physics and Institute of Physics, \\ Hunan Normal University, Changsha, Hunan 410081, China \\ ${ }^{2}$ Key Laboratory of Low Dimensional Quantum Structures \\ and Quantum Control of Ministry of Education, \\ Hunan Normal University, Changsha 410081
}

\begin{abstract}
The Chaplygin gas (CG) and the generalized Chaplygin gas (GCG) models, proposed as candidates of the unified dark matter-dark energy (UDME), are tested with the look-back time (LT) redshift data. We find that the LT data only give a very weak constraint on the model parameter. However, by combing the LT with the baryonic acoustic oscillation peak, we obtain, at the $95.4 \%$ confidence level, $0.68 \leq A_{c} \leq 0.82$ and $0.59 \leq h \leq 0.65$ for the CG model, and $0.67 \leq A_{s} \leq 0.89$ and $-0.29 \leq \alpha \leq 0.61$ for the GCG model. This shows that both the CG and the GCG are viable as a candidate of UDME. Within the GCG model, we also find that the Chaplygin gas model $(\alpha=1)$ is ruled out by these data at the $99.7 \%$ confidence level.

PACS numbers: 98.80.-k; 95.36.+x
\end{abstract}

\footnotetext{
* Corresponding author
} 


\section{INTRODUCTION}

The fact that the universe is undergoing an accelerating expansion has been supported by many astrophysical and cosmological observations, such as Type Ia Supernovae (Sne Ia) 11] and cosmic microwave background radiation (CMBR) [2, 3], etc. In order to explain this mysterious phenomenon, one usually assumes the existence of an exotic energy with negative pressure, named dark energy, which dominates the total energy density and causes an accelerating expansion of our universe at late times. So far many candidates have been proposed for dark energy, such as the quintessence [4], phantom [5], quintom [6], holographic [8] and agegraphic [9] models, et al.

In 2001 Kamenshchik et al. [10] proposed an interesting model of dark energy, named the Chaplygin gas (CG), for which the equation of state has the form

$$
p_{c g}=-\frac{A}{\rho_{c g}},
$$

where $\mathrm{A}$ is a positive constant. Inserting the above equation into the energy conservation equation, one can obtain the following expression for the CG energy density

$$
\rho_{c g}=\rho_{c g 0}\left(A_{c}+\frac{1-A_{c}}{a^{6}}\right)^{\frac{1}{2}}
$$

where $\rho_{c g 0}$ is the present energy density of the CG and $A_{c} \equiv \frac{A}{\rho_{c g 0}}$. Because the energy density evolution behaves between dark-matter-like $\left(\rho_{c g} \propto a^{-3}\right)$ at early times and darkenergy-like $\left(\rho_{c g}=-p_{c g}\right)$ at late times, the CG model has been proposed as a model of the unified dark matter and dark energy (UDME). For $A_{s}=0$, the CG behaves always like matter while for $A_{s}=1$ it behaves always like a cosmological constant.

Later Bento et al. [11] generalized the CG model by adding a new parameter in the equation of state:

$$
p_{g c g}=-\frac{A}{\rho_{g c g}^{\alpha}} .
$$

Here $\alpha$ is also a positive constant and $\alpha=1$ corresponds to the CG model. This generalized model was called the generalized Chaplygin gas (GCG). Combining the equation of state and the energy conservation equation for the GCG, we have

$$
\rho_{g c g}=\rho_{g c g 0}\left(A_{s}+\frac{1-A_{s}}{a^{3(1+\alpha)}}\right)^{\frac{1}{1+\alpha}}
$$


where $\rho_{g c g 0}$ is the present energy density of the GCG and $A_{s} \equiv A / \rho_{g c g 0}^{1+\alpha}$. Again, the density evolution in this model changes from $\rho_{g c g} \propto a^{-3}$ at early times to $\rho_{g c g}=$ constant at late times. As a result, the GCG has also been proposed as the UDME. Apparently, for $\alpha=0$ the GCG model behaves like the cold dark matter plus a cosmological constant, while for $A_{s}=0$, it behaves always like matter and for $A_{s}=1$, it behaves always like a cosmological constant.

Currently, works have been done on the CG and the GCG models [12] and many authors have attempted to constrain them by using various observational data, such as Sne Ia [13, 14, 15, 16, 17, 18], the CMBR [18, 19, 20], the gamma-ray bursts [21], the gravitational lensing [15, 17, 22], the X-ray gas mass fraction of clusters [14, 15, 16],

the large scale structure [18, 23], the age of high-redshift objects [24], and the Hubble parameter versus redshift [25].

Recently, based on the age of the old objects and the age of the universe from the WMAP5 [3], the lookback time-redshift (LT) data have been used to test different dark energy models [26]. The advantage of this data set is that the ages of distant objects are independent of each other, and thus may avoid biases that are present in techniques that use distances of primary or secondary indicators in the cosmic distance ladder method. As a result, these age data are different from the widely used distance one. Therefore, it may still be interesting to test the CG and the GCG models with these LT data although many other datasets have been used to test them. This is what we are going to do in the present paper. In order to break the degeneracy of model parameters, we also add, in our discussion, the baryonic acoustic oscillation (BAO) peak detected by large-scale correlation function of luminous red galaxies from Sloan Digital Sky Survey (SDSS) [27].

\section{THE LOOKBACK TIME-REDSHIFT DATA}

The lookback time as a function of redshift can be expressed as the interval from the age at redshift $z\left(t_{z}\right)$ to the present age of the Universe $\left(t_{0}\right)$,

$$
t_{L}(z ; \mathbf{p})=H_{0}^{-1} \int_{0}^{z} \frac{d z^{\prime}}{\left(1+z^{\prime}\right) E(\mathbf{p})}
$$


where $H_{0}=h / 9.78 \mathrm{Gry}^{-1}$ is the present value of the Hubble parameter and $h$ can be obtained from the HST key project (at $1 \sigma$ interval $0.64 \leq h \leq 0.80$ [28]). In the above expression, the form of $E(\mathbf{p})$ is dependent on the selected cosmological model

$$
E^{2}(\mathbf{p})=\sum_{i} \Omega_{i, 0} a^{-3\left(1+w_{i}\right)}
$$

where $E(\mathbf{p}) \equiv H(\mathbf{p}) / H_{0}$ and $\mathbf{p}$ represents the complete set of parameters for a given cosmological model with the subscript 0 denoting the present-day value.

The age $t$ at redshift $z_{i}$ of an object is defined as the difference between the age of the universe at redshift $z_{i}$ and that at redshift $z_{F}$ when the object was born

$$
t\left(z_{i}\right)=\frac{1}{H_{0}}\left[\int_{z_{i}}^{\infty} \frac{d z^{\prime}}{\left(1+z^{\prime}\right) E(\mathbf{p})}-\int_{z_{F}}^{\infty} \frac{d z^{\prime}}{\left(1+z^{\prime}\right) E(\mathbf{p})}\right] .
$$

Using the above expression and Eq. (5), $t\left(z_{i}\right)$ can also be written as

$$
t\left(z_{i}\right)=t_{L}\left(z_{F}\right)-t_{L}\left(z_{i}\right)
$$

The observed lookback time to an object at redshift $z_{i}$ can then be defined from the above relation [33]

$$
\begin{aligned}
t_{L}^{o b s}\left(z_{i}\right) & =t_{L}\left(z_{F}\right)-t_{L}\left(z_{i}\right) \\
& =\left[t_{0}^{o b s}-t\left(z_{i}\right)\right]-\left[t_{0}^{o b s}-t_{L}\left(z_{F}\right)\right] \\
& =t_{0}^{o b s}-t\left(z_{i}\right)-\tau
\end{aligned}
$$

where $\tau$ means the time from Big Bang to the formation of the objcet, which is the so-called delay factor or incubation time, and $t_{0}^{\text {obs }}=13.7 \pm 0.2$ Gyr [29].

Using the differential age method, recently, Simon et al. [30] obtained 32 age data points of the passively evolving galaxies in the redshift interval $0.117<z<1.845$, which are shown in Fig. (1). In order to constrain the model parameters using these age data, we define the likelihood function

$$
\mathcal{L}_{\text {age }} \propto \exp \left[-\chi_{\text {age }}^{2}(z ; \mathbf{p}, \tau) / 2\right]
$$

where $\chi_{\text {age }}^{2}$ is relative to the LT sample:

$$
\chi_{\text {age }}^{2}=\sum_{i} \frac{\left[t_{L}\left(z_{i} ; \mathbf{p}\right)-t_{L}^{o b s}\left(z_{i} ; \tau\right)\right]^{2}}{\sigma_{T}^{2}}+\frac{\left[t_{0}(\mathbf{p})-t_{0}^{o b s}\right]}{\sigma_{t_{o}^{o b s}}^{2}} .
$$


In the above expression, $\sigma_{T}^{2} \equiv \sigma_{i}^{2}+\sigma_{t_{0}^{\text {obs }}}^{2}, \sigma_{i}$ is the uncertainty in the individual lookback time to the $i^{\text {th }}$ galaxy of our sample and $\sigma_{t_{0}^{o b s}}$ stands for the uncertainty on the total expansion age of the universe $\left(t_{0}^{\text {obs }}\right)$. Note that while the observed lookback time $\left(t_{L}^{\text {obs }}\left(z_{i} ; \tau\right)\right)$ is directly dependent on $\tau$, its theoretical value $\left(t_{L}\left(z_{i} ; \mathbf{p}\right)\right)$ is not. Furthermore, in principle, it must be different for each object in the sample. Thus the delay factor becomes a "nuisance" parameter, we use the following method to marginalize over it [31]

$$
\begin{aligned}
\tilde{\chi}^{2} & =-2 \ln \int_{0}^{\infty} d \tau \exp \left(-\frac{1}{2} \chi_{\text {age }}^{2}\right) \\
& =A-\frac{B^{2}}{C}+D-2 \ln \left[\sqrt{\frac{\pi}{2 C}} \operatorname{erfc}\left(\frac{B}{\sqrt{2 C}}\right)\right],
\end{aligned}
$$

where

$$
A=\sum_{i=1}^{n} \frac{\triangle^{2}}{\sigma_{T}^{2}} . \quad B=\sum_{i=1}^{n} \frac{\triangle}{\sigma_{T}^{2}} . \quad C=\sum_{i=1}^{n} \frac{1}{\sigma_{T}^{2}},
$$

D is the second term of the rhs of Eq.(11),

$$
\triangle=t_{L}\left(z_{i} ; \mathbf{p}\right)-\left[t_{0}^{o b s}-t\left(z_{i}\right)\right]
$$

and $\operatorname{erfc}(\mathrm{x})$ is the complementary error function of the variable $\mathrm{x}$.

\section{CONSTRAINT ON THE CG MODEL}

\section{A. Constraint from lookback time-redshift test}

For the CG model, the Friedmann equation for a spatially flat universe which contains only the baryonic matter and the Chaplygin gas, can be expressed as

$$
H^{2}\left(H_{0}, A_{c}, h, z\right)=H_{0}^{2} E^{2}\left(A_{c}, z\right)
$$

with $E\left(A_{c}, z\right)$ being given by

$$
E\left(A_{c}, z\right)=\left[\Omega_{b}(1+z)^{3}+\left(1-\Omega_{b}\right)\left(A_{c}+\left(1-A_{c}\right)(1+z)^{6}\right)^{\frac{1}{2}}\right]^{1 / 2},
$$

where $\Omega_{b}$ is the present dimensionless density parameter of the baryonic matter and the WMAP observations give $\Omega_{b} h^{2}=0.0233 \pm 0.0008$ [3]. For LT data only, Fig. (2) shows the contour plot (68.3, 95.4 and $99.7 \%$ confidence level) in the $A_{c}-h$ plane for the $\chi_{a g e}^{2}$ given 
by Eqs. (10)-(12) with a Gaussian prior on the Hubble parameter. The best-fit value is $A_{c}=0.93$ and $h=0.74$ which agrees very well with the recent analysis of an extensive new program with the Hubble Space Telescope [34]. We obtain $0.32 \leq A_{c} \leq 0.98$ and $0.51 \leq h \leq 0.88$ at the $95.4 \%$ confidence level. Apparently, LT only gives a very weak constraint on the model parameter $A_{c}$

\section{B. Joint analysis with BAO}

In order to obtain a tighter constraint on the model parameter, we combine the LT data with the BAO data. For the BAO data, the parameter $\mathcal{A}$ is used, which is independent of cosmological models and for a flat Universe it can be expressed as

$$
\mathcal{A}=\frac{\sqrt{\Omega_{m}}}{E\left(z_{1}\right)^{1 / 3}}\left[\frac{1}{z_{1}} \int_{0}^{z_{1}} \frac{d z}{E(z)}\right]^{2 / 3}
$$

where $z_{1}=0.35$ and $\mathcal{A}$ is measured to be $\mathcal{A}=0.469 \pm 0.017$ from the SDSS. In the above expression, $\Omega_{m}$ is the effective matter density and it is given by $\Omega_{m}=\Omega_{b}+\left(1-\Omega_{b}\right)(1-$ $\left.A_{c}\right)^{1 / 2}$ [15, 16, 32] for the CG model as a UDME .

In Fig. (3), we show the results by combining the LT and BAO, which indicate that both $A_{c}$ and $h$ are tightly constrained. At $95.4 \%$ confidence level, we obtain $0.68 \leq A_{c} \leq 0.82$ and $0.59 \leq h \leq 0.65$ with the best value given by $A_{c}=0.74$ and $h=0.61$.

\section{CONSTRAINT ON THE GCG MODEL}

For the GCG model, $E(\mathbf{p})$ in the Friedmann equation now has the form

$$
\begin{aligned}
E\left(A_{s}, \alpha, z\right)= & {\left[\Omega_{b}(1+z)^{3}+\left(1-\Omega_{b}\right)\right.} \\
& \left.\times\left(A_{s}+\left(1-A_{s}\right)(1+z)^{3(1+\alpha)}\right)^{\frac{1}{1+\alpha}}\right]^{1 / 2} .
\end{aligned}
$$

Since we are interested in the parameters $A_{s}$ and $\alpha, h$ becomes a nuisance parameter and is fixed at $h=0.72$ in our discussion.

In Fig. (4), we show the results from the lookback time-redshift (LT) data, which is very similar to the results obtained from the Sne Ia data [13]. The best-fit values are 
$A_{s}=0.47$ and $\alpha=-0.85$. From this figure one can see that two model parameters $A_{s}$ and $\alpha$ are degenerate and at the $68.3 \%$ confidence level, the scenario of the standard dark energy (cosmological constant) plus dark matter scenario $(\alpha=0)$ is excluded.

The constraints from only the BAO data on the parameter space $A_{s}-\alpha$ shows that although it constrains efficiently the parameter plane into a narrow strip, parameters $A_{s}$ and $\alpha$ are also degenerate [25]. However, it is interesting to see that possible degeneracies between the model parameters may be broken by combining these two sets of observational data (LT+BAO). Fig. (5) shows the results in the $A_{s}-\alpha$ plane for the joint LT+BAO analysis. The best fit happens at $A_{s}=0.77$ and $\alpha=0.02$. At the $95.4 \%$ confidence level, we obtain a stringent constraint on the GCG: $0.67 \leq A_{s} \leq 0.89$ and $-0.29 \leq \alpha \leq 0.61$. Apparently, the Chaplygin gas model $(\alpha=1)$ is ruled out at the $99.7 \%$ confidence level. In addition, we find that, at the $68.3 \%$ confidence level, the combination of these two databases allows the scenario of the cosmological constant plus dark matter $(\alpha=0)$, although the lookback time-redshift alone excludes it.

\section{CONCLUSIONS}

In this paper, we have examined, with the look-back time redshift data, the constraints on the Chaplygin gas (CG) model and the generalized Chaplygin gas (GCG) model, which are proposed as candidates of the unified dark matter-dark energy (UDME). We find that, although the current lookback time-redshift data can not tightly constrain the CG and the GCG model parameters, stringent constraints can be obtained by combining the lookback time-redshift data with baryonic acoustic oscillation peak data. For the CG and the GCG models, at the $95.4 \%$ confidence level, we obtained $0.68 \leq A_{c} \leq 0.82$ and $0.59 \leq h \leq 0.65$, and $0.67 \leq A_{s} \leq 0.89$ and $-0.29 \leq \alpha \leq 0.61$, respectively. This shows that both the CG and the GCG are viable as candidates of UDME. However, from the results obtained within the GCG, we find that the Chaplygin gas model $(\alpha=1)$ is ruled out by these data at the $99.7 \%$ confidence level. These results are consistent with those obtained from the X-ray gas mass fractions of galaxy clusters and the dimensionless coordinate distance of Sne Ia and FRIIb radio galaxies [16], the CMBR power spectrum measurements from 
BOOMERANG and Archeops and the Sne Ia [20], and the Hubble parameter data and the Sne Ia [25].

\section{Acknowledgments}

This work was supported in part by the National Natural Science Foundation of China under Grants No. 10575035, 10775050, 10705055, the SRFDP under Grant No. 20070542002, the Programme for the Key Discipline in Hunan Province, the FANEDD under Grant No 200922 and the Research Fund of Hunan Provincial Education Department.

[1] A. G. Riess, et al., Astrophys. J. 116 (1998) 1009; A. G. Riess, et al., Astrophys. J. 607 (2004) 665; S. Perlmutter, et al., Astrophys. J. 517 (1999) 565; J. L. Tonry, et al., Astrophys. J. 594, 31 (2003) 1; B. J. Barris, et al., Astrophys. J. 602 (2004) 571; R. A. Knop, et al., Astrophys. J. 598 (2007) 102.

[2] A. Balbi, et al., Astrophys. J. 545 (2000) L1; P. de Bernarbis, et al., Nature 404 (2000) 955; A. H. Jaffe, et al., Phys. Rev. Lett. 86 (2001) 3475.

[3] D. N. Spergel, et al., Astorphys. J. Suppl. 148 (2003) 175; D. N. Spergel, et al.,Astrophys. J. Supl. 170 (2007) 377.

[4] C. Wetterich, Nucl. Phys. B 302, 668 (1988); B. Ratra and P. E. J. Peebles, Phys. Rev. D 37, 3406 (1988); R. Caldwell, R. Dave, and P. J. Steinhardt, Phys. Rev. Lett. 80, 1582 (1998).

[5] R. R. Caldwell, Phys. Lett. B 545, 23 (2002); R. R. Caldwell, M. Kamionkowski, and N. N. Weinberg, Phys. Rev. Lett. 91, 071301 (2003).

[6] B. Feng, X. Wang and X. Zhang, Phys. Lett. B 607, 35 (2005); B. Feng, M. Li, Y. Piao and X. Zhang, Phys. Lett. B 634, 101 (2006); Z. Guo, Y. Piao, X. Zhang and Y. Zhang, Phys. Lett. B 608, 177 (2005).

[7] H. Wei, R. G. Cai and D. F. Zeng, Class. Quant. Grav. 22, 3189 (2005); H. Wei and R. G. Cai, Phys. Rev. D 72, 123507 (2005); H. Wei, N. N. Tang and S. N. Zhang, Phys. Rev. D 
75, 043009 (2007).

[8] A. Cohen, D. Kaplan, A. Nelson, Phys. Rev. Lett. 82 (1999) 4971; S.D.H. Hsu, Phys. Lett. B 594 (2004) 13; M. Li, Phys. Lett. B 603 (2004) 1.

[9] R. Cai, Phys.Lett. B 657, 228-231 (2007); H. Wei and R. Cai, Phys. Lett. B 655, 1-6 (2007); H. Wei and R. Cai , Phys. Lett. B 660, 113-117 (2008).

[10] A. Kamenshchik, U. Moschella, V. Pasquier, Phys. Lett. B 511 (2001) 265.

[11] M. C. Bento, O. Bertolami, A. A. Sen, Phys. Rev. D 66 (2002) 043507.

[12] A. A. Sen, Robert J. Scherrer, Phys. Rev. D 72 (2005) 063511; R. Herrera, Gen.Rel.Grav. 41 (2009) 1259; S. Li, Y. Ma and Y. Chen, arXiv:0809.0617; S. del Campo and R. Herrera, Phys. Lett. B 665 (2008) 100; R. Herrera, Phys. Lett. B 664 (2008) 149; M. Jamil and M. A Rashid, Eur. Phys. J. C 58 (2008) 111; M. Jamil and M. A Rashid, Eur. Phys. J. C 60 (2009) 141; S. del Campo and R. Herrera, Phys. Lett. B 660 (2008) 282; P. Wu and H. Yu, JCAP 0703 (2007) 015; Class. Quant. Grav. 24 (2007) 4661; V. Gorini, A. Y. Kamenshchik, U. Moschella, O. F. Piattella and A. A. Starobinsky, JCAP 0802 (2008) 016; C. S. J. Pun, L. a. Gergely, M. K. Mak, Z. Kovacs, G. M. Szabo and T. Harko, Phys. Rev. D 77 (2008) 063528; J. Lu, L. Xu, Y. Gui, B. Chang, arXiv:0812.2074; X. Zhang, J. Zhang, J. Cui and L. Zhang, Mod. Phys. Lett. A 24 (2009) 1763; P. Thakur, S. Ghose and B. C.Paul, arXiv:0905.2281; O. F. Piattella, arXiv: 0906.4430; Y. Urakawa and T. Kobayashi, arXiv:0907.1191

[13] P. Wu and H. Yu, Astrophys. J. 658 (2007) 663; J. C. Fabris, S. V. B. Goncalves, P. E. de Souza, astro-ph/0207430; M. Makler, S. Q. Oliveira, I. Waga, Phys. Lett. B 555 (2003) 1; R. Colistete Jr., et al., Int. J. Mod. Phys. D 13 (2003) 669; O. Bertolami, A. A. Sen, S. Sen, P. T. Silva, Mon. Not. R. Astron. Soc. 353 (2004) 329; M.C. Bento, O. Bertolami, M.J. Rebouas, P.T. Silva, Phys. Rev. D73 (2006) 043504; X. Zhang, F. Wu, J. Zhang, JCAP 0601 (2006) 003.

[14] J. V .Cunha, J. A. S. Lima, J. S. Alcaniz, Phys. Rev. D 69 (2004) 083501.

[15] M. Makler, S. Q. Oliveira, I. Waga, Phys. Rev. D 68 (2003) 12.

[16] Z. H. Zhu, Astron. Astrophys. 423 (2004) 421.

[17] P. T. Silva, O. Bertolami, Astrophys. J. 599 (2003) 829. 
[18] R. Bean, O. Dore, Phys. Rev. D 68 (2003) 023515.

[19] M. C. Bento, O. Bertolami, A. A. Sen, Phys. Lett. B 575 (2003) 172; L. Amendola, L. F. Finelli, C. Burigana, D. Carturan, JCAP 0307 (2003) 005.

[20] M. C. Bento, O. Bertolami, A. A. Sen, Phys. Rev. D 67 (2003) 063003.

[21] O. Bertolami, P. T. Silva, Mon. Not. R. Astron. Soc. 365 (2006) 1149.

[22] A. Dev, D. Jain, J. S. Alcaniz, Phys. Rev. D 67 (2003) 023515; A. Dev, D. Jain, J. S. Alcaniz, Astron. Astrophys. 417 (2004) 847; D. M. Chen, Astrophys. J. 587 (2003) L55; D. M. Chen, Astron. Astrophys. 397 (2003) 415.

[23] N. Bilic, G. B. Tupper, R. D. Viollier, Phys. Lett. B 535 (2002) 17; R. C. Santos, J. F. Jesus, arXiv:0806.2822; T. Multamäki, M. Manera, E. Gaztanaga, Phys. Rev. D 69 (2004) 023004 .

[24] J. S. Alcaniz, D. Jain, A. Dev, Phys. Rev. D 67 (2003) 043514.

[25] P. Wu, H. Yu, Phys. Lett. B 644 (2007) 16-19.

[26] L. Samushia, A. Dev, D. Jain and B. Ratra, arXiv:0906.2734; S. Capozziello, et al., Phys. Rev. D 70 (2004) 123501; N. Pires, Z. Zhu and J. Alcaniz Phys. Rev. D 73 (2006) 123530; Z. L. Yi and T. Zhang, Mod. Phys. Lett. A 22 (2007) 41; M. A. Dantas and J. S. Alcaniz, arXiv:0901.2327; M. A. Dantas, J. S. Alcaniz, D. Jain and A. Dev, Astron. Astrophys. 467 (2007) 421.

[27] D. J. Eienstein, et al., Astrophys. J. 633 (2005) 560.

[28] W. Freedman, et al., Astrophys. J. 553 (2001) 47.

[29] J. Dunkley, et al., Astrophys. J. Suppl. 180 (2009) 306.

[30] J. Simon, L. Verde, and R. Jimenez, Phys. Rev. D 71 (2005) 123001

[31] M. A. Dantas, J. S. Alcaniz, D. Jain, A.Dev, Astron. Astrophys. 467 (2007) 421.

[32] M. C. Bento, O. Bertolami, A. A. Sen, Phys. Rev. D 70 (2004) 083519; J. A. S. Lima, J. V. Cunha, J. S. Alcaniz, astro-ph/0611007.

[33] S. Capozziello, et al., Phys. Rev. D 70 (2004) 123501; N. Pires, Z. H. Zhu, J. S. Alcaniz, Phys. Rev. D 73 (2006) 123530.

[34] Riess et al., Astrophys. J. 699 (2009) 539. 


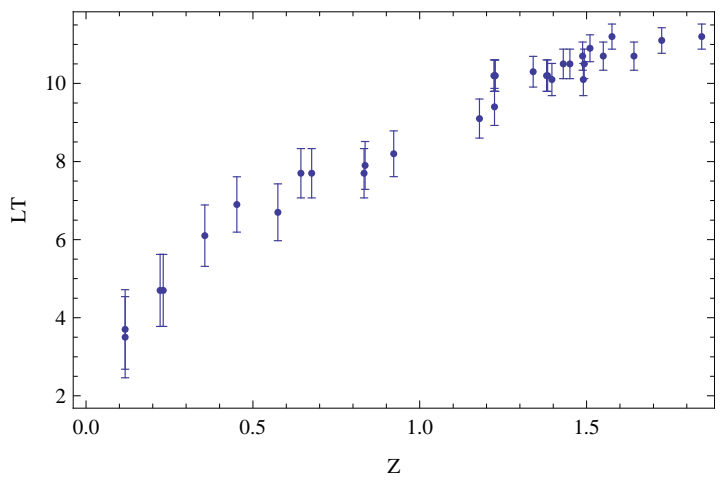

FIG. 1: The 32 look-back time redshift (LT) data points obtained from the old passive galaxies distributed over the redshift interval $0.11 \leq z \leq 1.84$ with the estimate of the total age of universe $t_{0}^{\text {obs }}=13.7 \pm 0.2$ Gry from the CMB data [29].

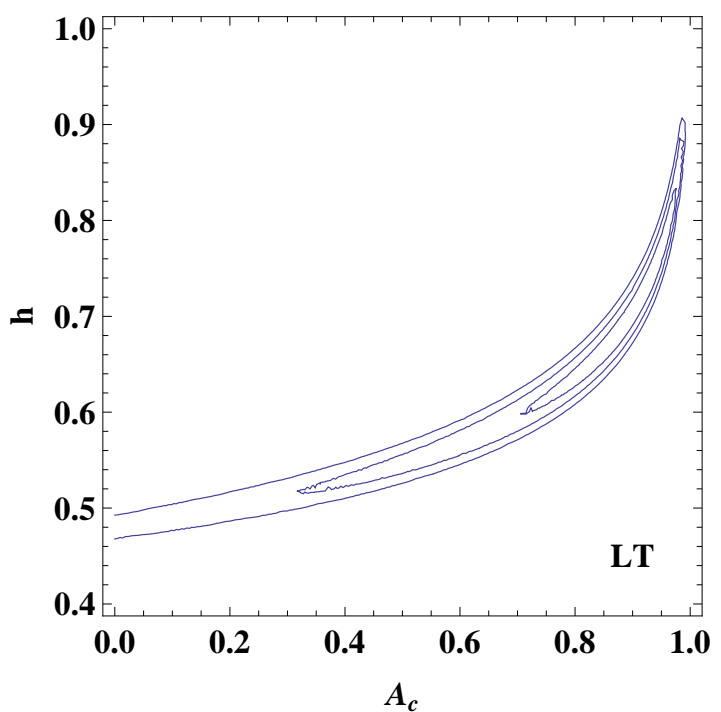

FIG. 2: The $68.3,95.4$ and $99.7 \%$ confidence level contours for $A_{c}$ versus $h$ from the lookback time-redshift data. The best fit happens at $A_{c}=0.77$ and $h=0.74$. 


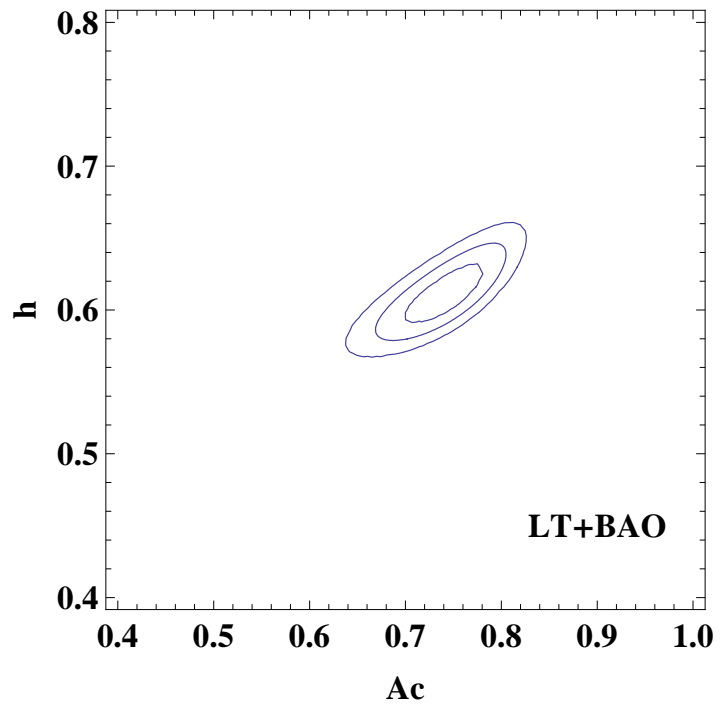

FIG. 3: The 68.3, 95.4 and $99.7 \%$ confidence level contours for $A_{c}$ versus $h$ from the lookback time-redshift data plus SDSS baryonic acoustic oscillations peak. The best fit happens at $A_{c}=$ 0.74 and $h=0.61$.

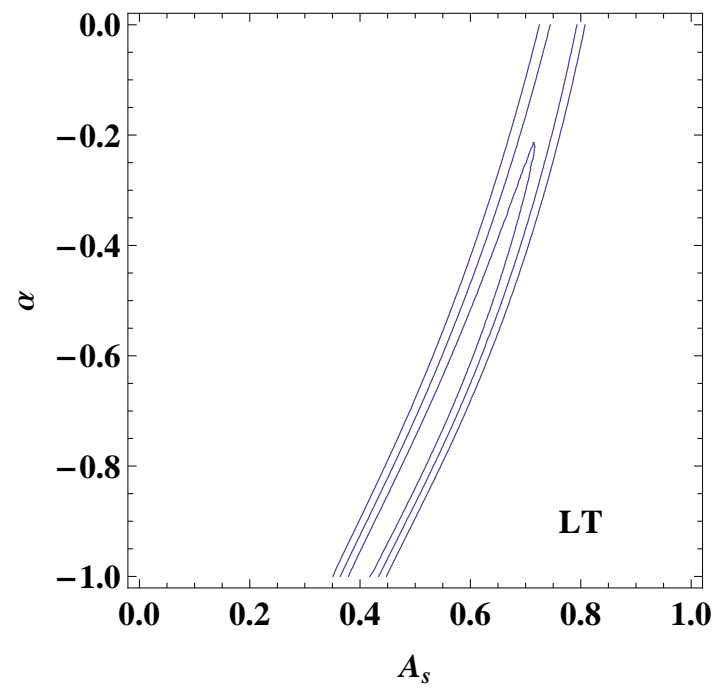

FIG. 4: The 68.3, 95.4 and $99.7 \%$ confidence level contours for $A_{s}$ versus $\alpha$ from the lookback time-redshift data with a fixed $\mathrm{h}=0.72$. The best fit happens at $A_{s}=0.47$ and $\alpha=-0.85$. 


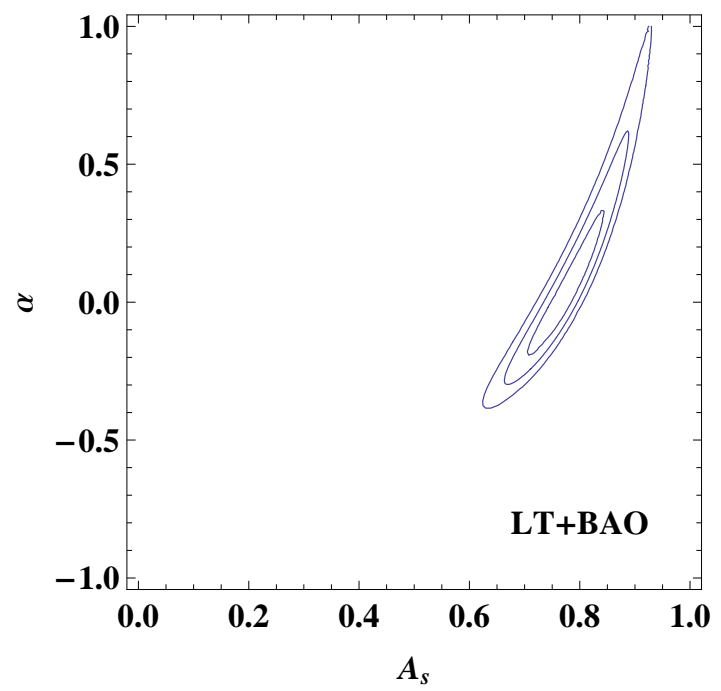

FIG. 5: The 68.3, 95.4 and $99.7 \%$ confidence level contours for $A_{s}$ versus $\alpha$ from the lookback time-redshift data plus SDSS baryonic acoustic oscillations peak. The best fit happens at $A_{s}=$ 0.77 and $\alpha=0.02$. 